\title{
Clareamento Dental - Verificação da Eficácia, Estabilidade de Cor e Nível de Sensibilidade
}

Tooth Bleaching - Evaluation of Effectiveness, Color Stability and Sensitivity Level

Blanqueamiento Dental - Comprobación de la Eficacia, la Estabilidad del Color y el Nivel de Sensibilidad

Jefferson Lucas MENDES

Graduando em Odontologia pela Universidade Estadual da Paraíba (UEPB), Araruna- PB, Brasil Brenno Anderson Santiago DIAS

Graduando em Odontologia pela Universidade Estadual da Paraíba (UEPB), Araruna- PB, Brasil Geovanna Caroline Brito da SILVA Graduanda em Odontologia pela Universidade Estadual da Paraíba (UEPB), Araruna- PB, Brasil Layla Narrely Santos ALVES Graduanda em Odontologia pela Universidade Estadual da Paraíba (UEPB), Araruna- PB, Brasil Francielly de Lemos MEDEIROS Graduanda em Odontologia pela Universidade Estadual da Paraíba (UEPB), Araruna- PB, Brasil Marcelo Gadelha VASCONCELOS Professor Doutor do Departamento de Odontologia da Universidade Estadual da Paraíba - UEPB, 58429-500, Araruna - PB, Brasil https://orcid.org/0000-0003-0396-553X Rodrigo Gadelha VASCONCELOS

Professor Doutor do Departamento de Odontologia da Universidade Estadual da Paraíba - UEPB, 58429-500, Araruna - PB, Brasil http://orcid.org/0000-0002-7890-8866

\section{Resumo}

Introdução: O clareamento dental consiste em um método simples, seguro e eficaz, frequentemente solicitado por pacientes que procuram melhorias estéticas. Dentre as formas de execução, estão disponíveis a técnica caseira, em consultório ou, ainda, a combinação de ambas. Objetivo: Verificar, através de uma revisão da literatura integrativa, quais técnicas e protocolos propiciaram melhor eficácia e estabilidade de cor, bem como as limitações e efeitos colaterais relacionados aos diferentes protocolos. Metodologia: Foi realizada uma revisão da literatura, por meio de buscas nas bases de dados PubMED/Medline, BVS e Scielo, utilizando os seguintes descritores: Tooth Bleaching, Hydrogen Peroxide, Bleaching Agents e Dentin Sensitivity. Foram incluídos estudos experimentais, publicados nos últimos cinco anos, com disponibilidade do texto de forma integral e clareza no detalhamento metodológico utilizado. Resultados: No método caseiro, o peróxido de hidrogênio ( $\mathrm{PH}$ ), em concentrações entre $4 \%$ e $10 \%$, e o peróxido de carbamida (PC), entre $10 \%$ e $37 \%$, demonstrou eficácia significativa no clareamento. Já o método de consultório isolado ou prévio ao tratamento caseiro, promoveu efeito clareador em uma ou duas sessões com aplicações que variaram entre 15 e 40 minutos, com $\mathrm{PH}$ em concentrações de até $38 \%$. Géis com menores concentrações provocaram menos sensibilidade dentária e irritação gengival, porém, nem sempre a cor desejada foi obtida. Conclusão: Para um tratamento eficaz é indispensável o conhecimento por parte do profissional em relação ao correto diagnóstico. Portanto, é fundamental conhecer a etiologia da mancha para assim estabelecer, de forma adequada, o tipo do gel, a sua concentração, tempo e modo de aplicação.

Descritores: Clareamento Dental; Peróxido de Hidrogênio; Clareadores; Sensibilidade da Dentina.

\section{Abstract}

Introduction: Tooth whitening is a simple, safe and effective method, frequently requested by patients looking for aesthetic improvements. Among the forms of execution, homemade technique is available, in the office or, even, the combination of both. Objective: To verify, through a review of the integrative literature, which techniques and protocols provided the best efficacy and color stability, as well as the limitations and side effects related to the different protocols. Methodology: A literature review was carried out by searching the PubMED / Medline, VHL and Scielo databases, using the following descriptors: Tooth Bleaching, Hydrogen Peroxide, Bleaching Agents and Dentin Sensitivity. Experimental studies, published in the last five years, were included, with full text availability and clarity in the methodological details used. Results: In the home method, hydrogen peroxide (PH), in concentrations between $4 \%$ and $10 \%$, and carbamide peroxide (PC), between $10 \%$ and $37 \%$, demonstrated significant efficacy in whitening. The isolated office method or prior to home treatment, however, promoted a lightening effect in one or two sessions with applications that varied between 15 and 40 minutes, with $\mathrm{PH}$ in concentrations of up to $38 \%$. Gels with lower concentrations caused less tooth sensitivity and gingival irritation; however, the desired color was not always obtained. Conclusion: For an effective treatment, knowledge on the part of the professional regarding the correct diagnosis is essential. Therefore, it is essential to know the etiology of the stain in order to establish the type of gel, its concentration, the time and the appropriate application method.

Descriptors: Tooth Bleaching; Hydrogen Peroxide; Bleaching Agents; Dentin Sensitivity.

\section{Resumen}

Introducción: El blanqueamiento dental es un método simple, seguro y efectivo, frecuentemente solicitado por pacientes que buscan mejoras estéticas. Entre las formas de ejecución se encuentran la técnica casera, en el consultorio o incluso una combinación de ambas. Objetivo: Verificar, a través de una revisión integrativa de la literatura, qué técnicas y protocolos proporcionaron mayor eficacia y estabilidad del color, así como las limitaciones y efectos secundarios relacionados con los diferentes protocolos. Metodología: Se realizó una revisión bibliográfica mediante búsqueda en las bases de datos PubMED/Medline, BVS y Scielo, utilizando los siguientes descriptores: Tooth Bleaching, Hydrogen Peroxide, Bleaching Agents y Dentin Sensitivity. Se incluyeron estudios experimentales publicados en los últimos cinco años, con disponibilidad de texto completo y claridad en el detalle metodológico utilizado. Resultados: En el método casero, el peróxido de hidrógeno (PH), en concentraciones entre $4 \%$ y $10 \%$, y el peróxido de carbamida (PC), entre $10 \%$ y $37 \%$, mostraron una eficacia significativa en la decoloración. Por otro lado, el método de consultorio aislado o previo al tratamiento domiciliario, promovió un efecto blanqueador en una o dos sesiones con aplicaciones que variaron entre 15 y 40 minutos, con pH en concentraciones de hasta $38 \%$. Los geles con concentraciones más bajas causaron menor sensibilidad dental e irritación gingival, sin embargo, no siempre se obtuvo el color deseado. Conclusión: Para un tratamiento eficaz es fundamental el conocimiento por parte del profesional en relación al correcto diagnóstico. Por ello, es fundamental conocer la etiología de la mancha para establecerla adecuadamente el tipo de gel, su concentración, tiempo y modo de aplicación.

Descriptores: Blanqueamiento de Dientes; Peróxido de Hidrógeno; Blanqueadores; Sensibilidad de la Dentina.

INTRODUÇÃO

A cor da estrutura dentária é um fator que influencia sobremaneira na estética e autoestima dos pacientes, tornando 0 tratamento de clareamento dental um dos procedimentos mais comuns na prática $\begin{array}{ll}\text { odontológica }^{1} \text { Apesar de um sorriso } \\ \text { esteticamente } & \text { agradável necessitar da }\end{array}$ contribuição de muitos outros fatores, tais como anatomia e boa oclusão, a cor do dente tem papel essencial, pois é rapidamente percebida em relação às outras alterações dentárias ${ }^{2}$. 
Mudanças na coloração dentária podem ocorrer devido à diversos fatores, como as condições do ambiente oral, a dieta, a higiene bucal e hábitos deletérios. Estes, são agrupados em fatores extrínsecos, passível de remoção por limpeza, e intrínsecos, onde a remoção quase sempre ocorre por clareamento dental ${ }^{3}$.

Desse modo, o clareamento dental configura-se como um procedimento seguro e amplamente utilizado que é frequentemente solicitado por pacientes que procuram melhorias estéticas $^{4}$. Trata-se de uma técnica de fácil execução, baixo custo, com bons resultados e menos invasiva quando comparado as restaurações adesivas diretas, por exemplo. Além disso, há uma gama de técnicas e protocolos com diferentes marcas, concentrações e aplicações que podem ser empregadas pelo cirurgião-dentista ${ }^{5}$.

Dentre as técnicas atualmente disponíveis para uso clínico estão o clareamento caseiro supervisionado pelo profissional, o clareamento realizado em consultório, ou, ainda, a associação entre essas duas técnicas ${ }^{3}$. No protocolo realizado em consultório a concentração do agente clareador, geralmente, é significativamente maior (20\% a $38 \%)$, já aplicações caseiras costumam empregar géis com concentrações entre $3 \%$ e $22 \%{ }^{6}$. Ambas as técnicas são usualmente realizadas com géis contendo peróxido de hidrogênio $(\mathrm{PH})$ e/ou peróxido de carbamida (PC) em diferentes concentrações.

Em relação ao mecanismo de ação desses produtos, pode-se dizer que o PC se decompõe em $\mathrm{PH}$ e ureia que, por sua vez, converte-se em amônia e $\mathrm{CO}_{2}$. Sendo assim, o $\mathrm{PH}$ é considerado o agente ativo, enquanto a ureia exerce função importante na elevação do $\mathrm{pH}$ da solução, contribuindo para efetivação do clareamento e proteção da estrutura dentária. Já o $\mathrm{PH}$ produz água, oxigênio e radicais livres, estes últimos subprodutos causam 0 efeito clareador ${ }^{6}$. Clinicamente, tal mecanismo é importante, pois o $\mathrm{PH}$ penetra no tecido dentário mais rápido do que o $\mathrm{PC}$, que precisa se dissociar em $\mathrm{PH}$ e ureia. Portando, o $\mathrm{PH}$ quando em contato com o dente, começa imediatamente o processo de clareamento pela liberação de oxigênio ${ }^{7}$.

Além de poder alterar o tipo de agente clareador, um tratamento eficaz pode variar a concentração do gel, tempo de aplicação, apresentação do produto, modo de aplicação e ativação ou não pela $\operatorname{luz}^{8}$.É responsabilidade do cirurgião dentista diagnosticar e estabelecer, de acordo com cada caso, o protocolo clínico a ser seguido ${ }^{2}$. Além de assegurar bons resultados clínicos, os profissionais buscam reduzir os efeitos indesejáveis durante e após o tratamento, sendo a sensibilidade dentária o efeito colateral mais relatado ${ }^{10}$.

Pacientes com histórico de sensibilidade dentária tem maior probabilidade de desenvolver hipersensibilidade pós-operatória ao procedimento, podendo estar associado a presença de retrações gengivais, trincas, desgaste de esmalte e baixo limiar de dor. Para mais, outro efeito colateral comum é a irritação gengival, pois as altas, e até mesmo as baixas concentrações de peróxidos podem provocar queimaduras e ulcerações nos tecidos moles do paciente. Dessa forma, quando realizado em consultório, sempre se deve fazer uso do isolamento absoluto ou de resinas fotoativadas que atuam como barreira gengival ${ }^{11}$.

Além de buscar técnicas $\mathrm{e}$ procedimentos que diminuam ou eliminem os efeitos supracitados, a durabilidade e estabilidade do tratamento clareador são fundamentais para sua eficácia ${ }^{12}$. A literatura é controversa em relação a recidiva de cor e estabilidade ao longo do tempo, nos dentes tratados com diferentes técnicas de clareamento $^{13}$. Diante do exposto, o objetivo deste estudo foi verificar, através de uma revisão da literatura integrativa, quais técnicas e protocolos propiciaram melhor eficácia e estabilidade de cor, bem como quais foram as limitações e efeitos colaterais relacionados aos diferentes protocolos de clareamento dental.

MATERIAL E MÉTODO

O presente estudo constituiu de uma revisão integrativa da literatura com o objetivo de sintetizar o conhecimento de maneira dinâmica sobre o tema proposto, de forma a transformá-lo em aplicação prática na clínica odontológica. A elaboração do estudo passou por 5 etapas metodológicas: formulação da pergunta que norteou o estudo, busca da literatura para compor a revisão, análise crítica dos estudos encontrados e incluídos na bibliografia, discussão dos resultados e confecção da revisão integrativa. Diante das diversas concentrações dos géis clareadores e dos muitos protocolos de aplicação dos mesmos, foram eleitas as seguintes perguntas para nortear o presente estudo: qual o melhor protocolo empregado nos clareamentos caseiro e de consultório? Qual destes apresentou maior estabilidade de cor ao longo do tempo e gerou menos reações adversas?

A coleta de dados foi realizada nas bases de dados eletrônicos: PubMED, BVS e Scielo, através do rastreio de artigos relevantes publicados no período de Janeiro de 2015 à 
Dezembro de 2020. Para escolha dos artigos, foram empregados os seguintes descritores: Clareamento dental (Tooth Bleaching), Peróxido de Hidrogênio (Hydrogen Peroxide), Clareadores (Bleaching Agents) e Sensibilidade da dentina (Dentin Sensitivity), foram utilizados os operadores booleanos "AND" e "OR" para filtragem dos artigos relacionados ao tema, utilizando as seguintes combinações: Clareamento dental AND Peróxido de hidrogênio OR Peróxido de carbamida AND Sensibilidade dental. Além disso, lançou-se mão de uma busca manual na lista de referência dos artigos selecionados, os resultados obtidos através da busca foram avaliados e classificados em elegíveis (estudos que apresentaram relevância e tinham possibilidade de ser incluídos na revisão) e não elegíveis (estudos sem relevância, sem possibilidade de inclusão na revisão).

Para escolha dos artigos foram utilizados critérios de inclusão que serviram como filtro para a seleção dos estudos: artigos publicados entre janeiro de 2015 e dezembro de 2020, disponibilidade do texto de forma integral nos idiomas português, inglês ou espanhol e clareza no detalhamento metodológico utilizado.

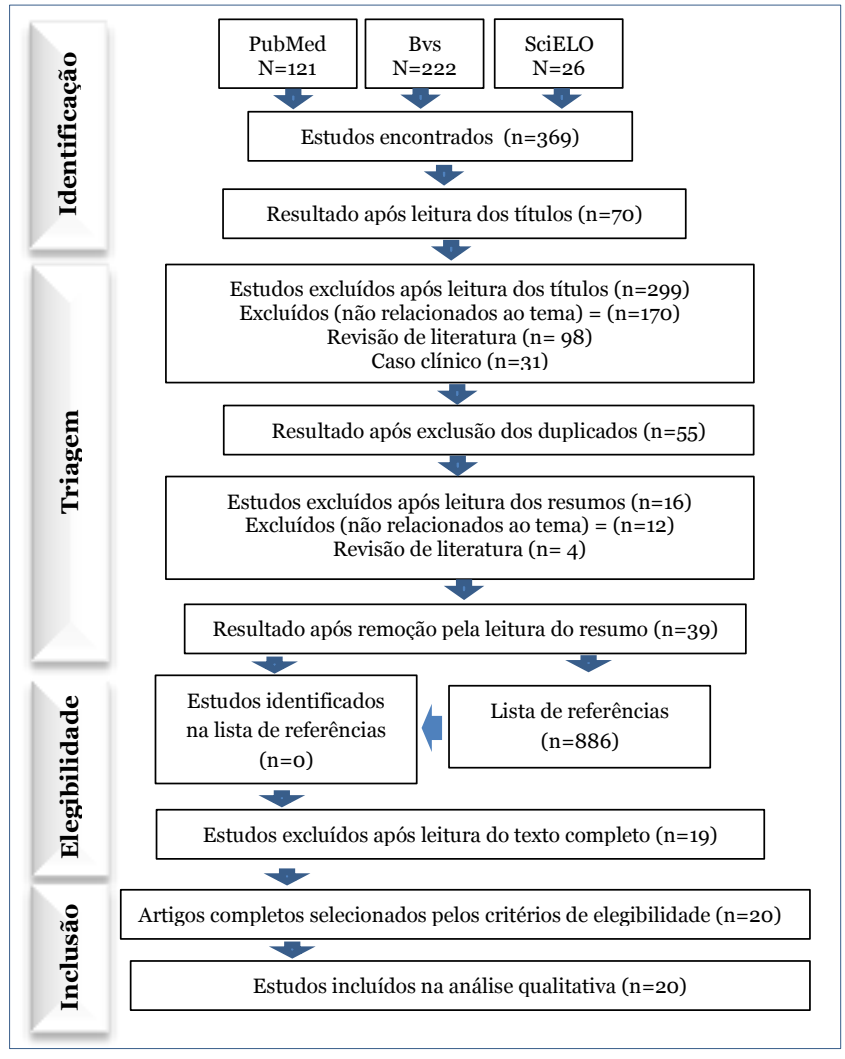

Figura 1. Fluxograma do processo de busca e seleção dos artigos.

Para exclusão dos artigos foram adotados como critérios: disponibilidade da publicação apenas em forma de resumo, trabalhos que não estivessem dentro dos requisitos estabelecidos na pesquisa (especificação do agente clareador utilizado e/ou clareza nos métodos e protocolos utilizados para o clareamento), pesquisas que utilizaram outro agente clareador que não $\mathrm{PH}$ e/ou PC, estudos em dentes não vitais, pesquisas com menos de 10 participantes ou não relacionados ao escopo de nosso estudo. $\mathrm{Na}$ figura 1 pode ser observado o processo de seleção dos artigos.

RESULTADOS

\section{- Características dos estudos}

Dos estudos selecionados, nove testaram a técnica do clareamento em consultório, seis realizaram o tratamento por meio da técnica caseira supervisionada e os cinco demais, por sua vez, analisaram os efeitos da associação de ambas as técnicas. Todos os pacientes dessas pesquisas realizaram 0 tratamento com aplicação em consultório ou caseiro com supervisão de um profissional. A tabela 1 apresenta um resumo das principais características e resultados encontrados nesses estudos.

Os achados de Kose et al. $^{14}$, ao analisarem diferentes protocolos para 0 clareamento de consultório com peróxido de hidrogênio $(\mathrm{PH})$ a $35 \%$, em cinquenta e três pacientes, evidenciaram que uma única aplicação de 15 minutos resulta em menores efeitos adversos, entretanto, diminui o nível de clareamento. O protocolo com 3 aplicações de 15 minutos resulta em um clareamento satisfatório, mas com alto índice de sensibilidade dentária (SD). Entretanto, o tratamento com duas aplicações resultou em clareamento similar ao do grupo com 3 aplicações, porém com menor nível de SD. Em contrapartida os estudos de Bacaksiz et al. ${ }^{15}$, asseguraram a eficácia do $\mathrm{PH}$ a $36 \%$ e $25 \%$ no clareamento dos dentes de pacientes jovens com 3 aplicações de 15 minutos em uma única sessão, sem ter ocorrido relato de qualquer efeito colateral.

Segundo Carlos et al. ${ }^{16}$, o tratamento clareador realizado com peróxido de carbamida (PC) a $10 \%$ e com $\mathrm{PH}$ a $10 \%$ e $9,5 \%$ foi mais eficaz quando realizado durante 14 dias, apresentando resultado superior e maior estabilidade de cor, quando comparado ao clareamento feito por 7 dias. Não houve diferenças significativas para os diferentes tipos de géis. Infere-se ainda que o uso mais prolongado não resultou em aumento de sensibilidade dentinária e outros efeitos adversos, pois ambos os grupos apresentaram sensibilidade dentinária leve e irritação gengival localizada. Em consonância, Chemin et al. ${ }^{17}$, apontaram que o clareamento caseiro usando $\mathrm{PH}$ a $10 \%$ é eficaz em 14 dias de tratamento. 
Tabela 1. Sumário das principais características dos estudos elegíveis para análise qualitativa (Fonte: Dados da pesquisa)

\section{Bacaksiz et al., 2016}

País

Tipo de clareamento/

Protocolo clínico

\begin{tabular}{l} 
\\
\hline Géis clareadores
\end{tabular}

Resultados

Efeitos adversos

Carlos et al., 2016 País

\section{6}

Não houve relato de efeitos adversos nos pacientes

Clareamento de consultório.

28 voluntários, com idades entre 13-18 anos, divididos aleatoriamente em dois grupos. $\mathrm{G} 1$ : $\mathrm{PH}$ a $25 \%$ com luz UV, sessão única com 3 aplicacỗes de 15 minutos e Gs: $\mathrm{PH}$ a $36 \% \mathrm{com}$ aplicaçoes de 15 minutos e G2: PH a $36 \%$ com luz LED em um único procedimento de $3 X$ por 15 minutos. A avaliação da cor foi feita com um espectrofômetro, antes e depois do clareamento; 48h, 1, 6 e 12 meses depois. A sensibilidade foi avaliada por meio de uma escala e posteriormente foi realizado o teste
estatico de Wilcoxon.

Zoom2: PH 25\%; Beaming White: PH 36\%. Ambos os grupos demonstraram a eficácia do clareamento. Não foi observado diferenças statisticamente significativas entre eles.

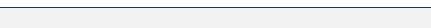

Brasil

Clareamento caseiro.

75 pacientes foram submetidos ao estudo, havendo aplicação de $\mathrm{PH}$ por $30 \mathrm{~min} / 14$ dias e PC por $8 \mathrm{~h} / 14$ dias. As avaliações foram realizadas no início do estudo, aos 7 dias e 14 dias pós-tratamento. A mudança de cor foi medida com a Commission internationale de l'e'clairage color coordinates, Vita Classical, e 3D Master scales. A escala visual analógica foi usada para avaliar a sensibilidade dentinária, aceitação do método e grau de conforto. Já a Irritação gengival foi avaliada como presente ou ausente e localizada ou generalizada.

\section{Géis clareadores}

PH a 10\% (Opalescence Go 10\%), PH a 9,5\% (Pola Day) e PC a 10\% (Opalescence PF 10\%).

Mudança de cor foi semelhante para todos os grupos em 7 dias e 14 dias, mas houve uma redução maior na tonalidade amarela em 14 dias. Irritação gengival localizada fo observada em ambos os métodos. Leve sensibilidade dentinária foi observada com o tempo, independentemente da concentração do agente clareador ou o tempo de aplicação.

\begin{tabular}{|l|l}
\hline Efeitos adversos & $\begin{array}{l}\text { Ambos os grupos apresentaram sensibilidade } \\
\text { dentinária leve e irritação gengival localizada. }\end{array}$ \\
\hline
\end{tabular}

Kose et al., 2016

País

Brasil

Clareamento de consultório.

53 pacientes distribuídos aleatoriamente em 53 pacientes distribuidos aleatoriamente em em um (1x15), dois (2x15) ou três (3x15)

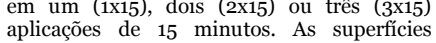
aplicações de 15 minutos. As superfícies
vestibulares dos dentes anteriores foram vestibulares dos dentes anteriores foram
submetidas ao clareamento. Duas sessões de clareamento, com uma semana de intervalo entre elas, foram realizadas. A avaliação de entre elas, foram realizadas. A avaliação de
cor foi realizada com uma guia visual e espectrofotômetro antes e 30 dias após o clareamento. Participantes expressaram a sensibilidade dentária com uma escala verbal de cinco pontos.

\begin{tabular}{|c|c|}
\hline Géis clareadores & PH a $35 \%$. \\
\hline Resultados & $\begin{array}{l}\text { Clareamento significativo foi observado em } \\
\text { todos os grupos, com clareamento } \\
\text { estatisticamente inferior para o grupo (15x1), } \\
\text { porém com menor nível de sensibilidade } \\
\text { dentária. O protocolo com } 2 \times 15 \text { produziu um } \\
\text { grau de clareamento semelhante ao do grupo } \\
\text { 3x15, mas com menor intensidade de SD. }\end{array}$ \\
\hline Efeitos adversos & $\begin{array}{l}3 \text { aplicações de } 15 \text { minutos em uma única } \\
\text { sessão resultaram no aumento do nível de } \\
\text { sensibilidade dentária. }\end{array}$ \\
\hline \multicolumn{2}{|l|}{ Machado et al., 2016} \\
\hline País & Brasil \\
\hline $\begin{array}{l}\text { Tipo de clareamento/ } \\
\text { Protocolo clínico }\end{array}$ & $\begin{array}{l}\text { Clareamento em consultório combinado com } \\
\text { o clareamento caseiro (G1); somente } \\
\text { clareamento caseiro (G2). G1: sessão prévia, } \\
\text { de } 15 \text { min, em consultório com PH a } 38 \% \text {, } \\
\text { seguida de aplicações caseiras do PC } \\
10 \% / 4 \mathrm{~h} / \text { dia/ } 14 \text { dias. G2: apenas aplicação } \\
\text { supervisionada com PC a } 10 \% \text {, } 4 \text { h dia, } \\
\text { durante } 14 \text { dias. }\end{array}$ \\
\hline Géis clareadores & $\begin{array}{l}\text { PH a 38\% (Opalescence Boost } 38 \% \text { PF, } \\
\text { Ultradent); PC a a 10\% (Opal-escence, } \\
\text { Ultradent). }\end{array}$ \\
\hline Resultados & $\begin{array}{l}\text { As duas técnicas promoveram clareamento } \\
\text { satisfatório e semelhantes alterações de cor. }\end{array}$ \\
\hline Efeitos adversos & $\begin{array}{l}\text { A técnica combinada demonstrou } \\
\text { clinicamente maiores índices de sensibilidade } \\
\text { dentária. }\end{array}$ \\
\hline
\end{tabular}

Tabela 1 (continuação). Sumário das principais características dos estudos elegíveis para análise qualitativa (Fonte: Dados da pesquisa) Rezende et al., 2016

País Brasil

Clareamento por meio da técnica combinada. 30 pacientes foram divididos, aleatoriamente, em dois grupos e submetidos a uma única sessão prévia de clareamento em consultório de 45 minutos com PH a $35 \%$ ou PH a $20 \%$. O

Tipo de clareamento/

Protocolo clínico clareamento caseiro foi realizado com PC $10 \%$ por duas horas diárias durante duas semanas. A cor foi avaliada com o guia de cores organizado pelo valor utilizando o Vita Clássico em diferentes períodos de até 12 meses após o clareamento.

Géis clareadores $\mathrm{PH}$ a $35 \%$ e $20 \%$; PC a $10 \%$.

A técnica combinada de clareamento usando A técnica combinada de clareamento usand clareamica conento

Resultados 12 meses, independentemente da concentração do PH usado para clareamento em consultório.

\begin{tabular}{l|l} 
& em consultório. \\
\hline Efeitos adversos & O protocolo com peróxido de hidrogênio a \\
$20 \%$ produziu menor risco e menor
\end{tabular}
$20 \%$ produziu menor risco e
intensidade de sensibilidade dentária.

Lima et al., 2018

País

Tipo de clareamento/

Protocolo clínico

Brasil

Clareamento de consultório.

25 pacientes foram aleatoriamente 25 pacientes foram aleatoriament $15 \%$ ou PH a $35 \%$. O agente clareador fo $15 \%$ ou PH três aplicaç̃es de 15 minutor por aplicado em três aplicações de 15 minutos po sessão. Dus sessoes de clareamento foram separadas por uma semana de interval.

Géis clareadores

Peróxido de hidrogênio a $35 \%$ e $15 \%$; grupo tratado com concentração a $35 \%$ apresentou clareamento significativament mais satisfatório.

Resultados

dentária maiore pintensidade de sensibilidade PH a $15 \%$.

Efeitos adversos

Loguercio et al., 2017 País com incisivo superior direito mais escuro que A2, foram selecionados. Os dentes foram clareados em duas sessões, com intervalo de semana. Cada sessão teve três aplicações de 8 min cada, de acordo com as instruções do fabricante. As mudanças de cor foram avaliadas por meio da Vita Classical e Vita Bleached guide, e métodos objetivo (espectrofotômetro de tonalidade fácil). Nível de sensibilidade dentária expressa com escala visual analógica.

Tipo de clareamento/
Protocolo clínico Gel ácido PH a $35 \%$ (Pola Office

Géis clareadores

Resultados SDI,Bayswater, Victoria); Gel neutro $\mathrm{PH}$ $37,5 \%$ (Pola Office SDI, Bayswater, Victoria). Todos os grupos alcançaram o mesmo nível de clareamento após 30 dias de avaliação clínica. O uso de um gel clareador neutro diminui significativamente o risco absoluto de sensibilidade dentária.

Efeitos adversos Géis clareadores ácidos apresentaram maior nível de sensibilidade dentária.

Pinto et al., 2017 País

\section{Brasil}

Clareamento caseiro. 30 pacientes foram submetidos ao clareamento a base de $\mathrm{PH}$,

Tipo de clareamento/ Protocolo clínico sendo divididos aleatoriamente em 4 grupos: (A) $\mathrm{PH}$ a $6 \%$, (B) $\mathrm{PH}$ a $7,5 \%$ (C) $\mathrm{PH}$ a $10 \%$ (D) Grupo controle-placebo. As avaliações foram realizadas antes do tratamento, bem como aos $7,30,180$ e 360 dias apos foram usados para análise de cor.

Géis clareadores

Peróxido de hidrogênio a 6\% (White Class with calcium - FGM), 7,5\% (White Class with calcium - FGM) e 10\% (Oral B 3D White).

Resultados Ambos os protocolos obtiveram clareamento no cicreivas entre eles. Nano houve melhora no clarean

Efeitos adversos

Vildósola et al., 2017 Relato de sensibilidade dentinária leve e desconforto, após $1^{\mathrm{a}}$ semana de tratamento.

País

Chile

Clareamento de consultório. 30 pacientes divididos em 2 grupos. Grupo A: 3 aplicações de 12 minutos para duas sessões; grupo B: aplicação de 36 minutos em duas sessões. A sessões foram marcadas com um intervalo de dois dias. A cor foi avaliada em cada sessão, bem como uma semana e um mês após bem como uma semana e um mês após Escala visual analógica foi utilizada para análise da sensibilidade dentária. Peróxido de hidrogênio a $6 \%$.

Peróxido de hidrogenio a 6\%. Ambos os protocolos obtiveram clareamento satisfatório, não havendo diferença significativas entre eles.

Sensibilidade leve relatada em ambos os

Resultados

Efeitos adversos grupos. 
Tabela 1 (continuação). Sumário das principais características dos estudos elegíveis para análise qualitativa (Fonte: Dados da pesquisa) Bersezio et al., 2018

\begin{tabular}{|c|c|}
\hline País & Chile \\
\hline $\begin{array}{l}\text { Tipo de clareamento/ } \\
\text { Protocolo clínico }\end{array}$ & $\begin{array}{l}\text { Clareamento de consultório. } 33 \text { pacientes } \\
\text { foram selecionados para o estudo. O } \\
\text { procedimento foi realizado seguindo o modelo } \\
\text { de "boca dividida". Um lado de cada boca foi } \\
\text { tratado aleatoriamente com PH em duas } \\
\text { diferentes concentracốes. Foram realizadas } \\
\text { duas sessóes com intervalo de uma semana } \\
\text { entre cada uma; a avaliação de cor foi por } \\
\text { meio de espectrofotômetro. }\end{array}$ \\
\hline Géis clareadores & $\begin{array}{l}\text { PH a 37,5\% (Polaoffice + 37,5\% SDI Limited); } \\
\text { PH a 6\% (Polaoffice +6\% SDI Limited). }\end{array}$ \\
\hline Resultados & $\begin{array}{l}\text { Ambos os protocolos demostraram eficácia. } \\
\text { Entretanto, houve diferença significativa no } \\
\text { efeito clareador, o gel de maior concentração } \\
\text { proporcionou melhores resultados. }\end{array}$ \\
\hline Efeitos adversos & $\begin{array}{l}\text { Relatos de sensibilidade dentária em ambos } \\
\text { os grupos, não havendo diferenças } \\
\text { significativas }\end{array}$ \\
\hline \multirow{2}{*}{\multicolumn{2}{|c|}{ Chemin et al., 2018}} \\
\hline País & \\
\hline $\begin{array}{l}\text { Tipo de clareamento/ } \\
\text { Protocolo clínico }\end{array}$ & $\begin{array}{l}\text { lareamento caseiro. } 78 \text { pacientes foram } \\
\text { submetidos a o tratamento, divididos em dois } \\
\text { grupos: (A) PH a } 4 \% \text { e (B) PH a 10\%. } 10 \% \text { PH } \\
\text { Aplicacóes por } 30 \text { minutos durante } 14 \text { dias. }\end{array}$ \\
\hline Géis clareadores & $\begin{array}{l}\text { PH 4\% (White Class } 4 \% \text {, FGM); } \\
\text { PH } 10 \% \text { (White Class } 10 \% \text {, FGM. }\end{array}$ \\
\hline Resultados & $\begin{array}{l}\text { O clareamento caseiro é éficaz com a } \\
\text { utilização de PH nas concentraçôes de } 4 \text { e } \\
\text { 10\%. Um mês após o clareamento, os dois } \\
\text { grupos apresentaram boa estabilidade de cor. }\end{array}$ \\
\hline Efeitos adversos & $\begin{array}{l}\text { O PH a } 10 \% \text { aumentou o risco absoluto e a } \\
\text { intensidade de sensibilidade dentinária. }\end{array}$ \\
\hline \multirow{2}{*}{\multicolumn{2}{|c|}{$\begin{array}{l}\text { Chemin et al., } 2018 \\
\text { País }\end{array}$}} \\
\hline & \\
\hline $\begin{array}{l}\text { Tipo de clareamento/ } \\
\text { Protocolo clínico }\end{array}$ & $\begin{array}{l}\text { Clareamento caseiro. } 20 \text { voluntários com } \\
\text { incisivos centrais A2 ou mais escuro foram } \\
\text { selecionados para este estudo. Foi utilizado } \\
\text { PH a } 10 \% \text { por trinta minutos, duas vezes ao } \\
\text { dia, durante duas semanas. }\end{array}$ \\
\hline Géis clareadores & Peróxido de hidrogênio a $10 \%$. \\
\hline Resultados & $\begin{array}{l}\text { Clareamento caseiro usando PH a } 10 \% \text { é } \\
\text { eficaz em } 14 \text { dias de clareamento. }\end{array}$ \\
\hline Efeitos adversos & Sensibilidade dentinária leve. \\
\hline \multicolumn{2}{|l|}{ Cordeiro et al., 2018} \\
\hline País & Brasil \\
\hline $\begin{array}{l}\text { Tipo de clareamento/ } \\
\text { Protocolo clínico }\end{array}$ & $\begin{array}{l}\text { Clareamento caseiro. } 60 \text { pacientes, com } \\
\text { incisivos superiores mais escuros do que A2 } \\
\text { foram selecionados para este estudo único- } \\
\text { cego, randomizado. Os dentes foram } \\
\text { clareados durante } 14 \text { dias com aplicaçóes de } \\
30 \text { minutos com o gel clareador, mudando } \\
\text { apenas a forma de aplicação. G1 (30): } \\
\text { moldeira convencional, G2 (30): moldeira } \\
\text { descartável pré-preenchida. }\end{array}$ \\
\hline Géis clareadores & PH a 10\% (White Class, FGM) \\
\hline Resultados & $\begin{array}{l}\text { Clareamento significativo foi observado em } \\
\text { todos os grupos após } 30 \text { dias de avaliação } \\
\text { clínica sem diferença significativa entre eles. }\end{array}$ \\
\hline Efeitos adversos & $\begin{array}{l}\text { Irritação gengival leve e transitória, e sem } \\
\text { diferença significativa observada entre oo } \\
\text { grupos, mesma situação para a sensibilidade } \\
\text { dentária. }\end{array}$ \\
\hline \multirow{2}{*}{\multicolumn{2}{|c|}{ Ferraz et al., 2018}} \\
\hline & Alemanha \\
\hline $\begin{array}{l}\text { Tipo de clareamento/ } \\
\text { Protocolo clínico }\end{array}$ & $\begin{array}{l}\text { Clareamento em consultório. } 54 \text { pacientes } \\
\text { submetidos ao clareamento, divididos em } 2 \\
\text { grupos: (A) PH } 6 \% \text { e (B) PH } 15 \% \text {. Foram } \\
\text { realizadas } 3 \text { aplicaçôes de } 10 \text { min. em cada } \\
\text { uma das } 2 \text { sessôes, com } 1 \text { semana de intervalo }\end{array}$ \\
\hline Géis clareadores & PH a 6\% e $15 \%$. \\
\hline Resultados & $\begin{array}{l}\text { Ambos os agentes apresentaram eficácia de } \\
\text { clareamento mas o PH a } 15 \% \text { apresentou } \\
\text { maior estabilidade de cor do que o PH a } 6 \% \text {, } \\
\text { aos } 6 \text { meses de acompanhamento. }\end{array}$ \\
\hline Efeitos adversos & $\begin{array}{l}\text { Os agentes causaram baixos níveis de } \\
\text { sensibilidade dentinária, irritação gengival e } \\
\text { não afetaram a qualidade relacionada à saúde } \\
\text { bucal de vida dos participantes. }\end{array}$ \\
\hline \multicolumn{2}{|l|}{ Martins et al., 2018} \\
\hline País & Colômbia \\
\hline $\begin{array}{l}\text { Tipo de clareamento/ } \\
\text { Protocolo clínico }\end{array}$ & $\begin{array}{l}\text { Clareamento em consultório. } 44 \text { pacientes } \\
\text { com caninos superiores direitos mais escuros } \\
\text { do que C2, foram selecionados para este } \\
\text { ensaio multicêntrico, simples-cego, } \\
\text { randomizado. O agente clareador foi aplicado } \\
\text { em } 2 \text { aplicaçôes de } 20 \text { min ou } 1 \text { apl/40 min } \\
\text { nas } 2 \text { sessões de tratamento. }\end{array}$ \\
\hline Géis clareadores & PH a $38 \%$. \\
\hline Resultados & $\begin{array}{l}\text { Aplicacacão } 40 \text { minutos do agente clareador } \\
\text { produziu o mesmo grau de clareamento e } \\
\text { sensibilidade dentária } 2 \text { aplicações } 20 \\
\text { minutos }\end{array}$ \\
\hline Efeitos adversos & $\begin{array}{l}\text { Presença de sensibilidade dentária com } \\
\text { intensidade equiparável nos dois grupos. }\end{array}$ \\
\hline
\end{tabular}

Tabela 1 (continuação). Sumário das principais características dos estudos elegíveis para análise qualitativa (Fonte: Dados da pesquisa) Vaez et al., 2018

\begin{tabular}{|c|c|}
\hline País & Brasil \\
\hline $\begin{array}{l}\text { Tipo de clareamento/ } \\
\text { Protocolo clínico }\end{array}$ & $\begin{array}{l}\text { Clareamento em consultório associado ao } \\
\text { caseiro; clareamento caseiro. } 26 \text { pacientes } \\
\text { usaram PC a } 10 \% \text { ih/dia até obter } \\
\text { clareamento satisfatório. Metade dos } \\
\text { participantes passou por uma sessão } \\
\text { preliminar de clareamento dental em } \\
\text { consultório com PH a } 35 \% \text { por } 45 \text { minutos. }\end{array}$ \\
\hline Géis clareadores & $\begin{array}{l}\text { PC a 10\% (Whiteness Perfect, FGM);PH a } \\
35 \% \text { (Whiteness HP Maxx, FGM). }\end{array}$ \\
\hline Resultados & $\begin{array}{l}\text { Sessão preliminar de consultório reduziu o } \\
\text { tempo necessario para obter a cor satisfatória } \\
\text { do dente em casa, porém aumentou o risco e o } \\
\text { nível de sensibilidade dentária. }\end{array}$ \\
\hline Efeitos adversos & $\begin{array}{l}\text { Relatos de sensibilidade dentária em ambos } \\
\text { os grupos, não havendo diferenças } \\
\text { significativas }\end{array}$ \\
\hline \multicolumn{2}{|l|}{ Zlataric et al., 2019} \\
\hline País & Croácia \\
\hline $\begin{array}{l}\text { Tipo de clareamento/ } \\
\text { Protocolo clínico }\end{array}$ & $\begin{array}{l}\text { Clareamento em consultório associado ao } \\
\text { caseiro. 30 pacientes foram divididos } \\
\text { aleatoriamente em dois grupos: G1 (15): os } \\
\text { pacientes receberam o tratamento associando } \\
\text { as duas técnicas. Sessão prévia em consultório } \\
\text { com PH a } 6 \%, 4 \text { aplicaçóes de } 15 \text { minutos. Em } \\
\text { seguida o clareamento caseiro foi realizado } \\
\text { por } 2 \text { semanas com PC a } 16 \% \text {, } 6 \mathrm{~h} / \text { dia. G2 } \\
\text { (15): grupo controle, pacientes passaram } \\
\text { pelos mesmos procedimentos com placebo. }\end{array}$ \\
\hline Géis clareadores & $\begin{array}{l}\text { PH a 6\% (Zoom2 kit; Philips Oral Healthcare, } \\
\text { Stamford, CT); PC a } 16 \% \text { (Polanight, SDI, } \\
\text { Melbourne, Australia). }\end{array}$ \\
\hline Resultados & $\begin{array}{l}\text { Aumento estatisticamente significativo na } \\
\text { mudança de cor com a aplicação clínica do } \\
\text { tratamento combinado }\end{array}$ \\
\hline Efeitos adversos & $\begin{array}{l}\text { Sem relatos de sensibilidade dentinária ou } \\
\text { irritação gengival. }\end{array}$ \\
\hline \multicolumn{2}{|l|}{ Estay et al., 2020} \\
\hline País & Chile \\
\hline $\begin{array}{l}\text { Tipo de clareamento/ } \\
\text { Protocolo clínico }\end{array}$ & $\begin{array}{l}\text { Clareamento em consultório. Grupo de } 25 \\
\text { pacientes foram submetidos a três aplicaçôes } \\
\text { do gel por } 12 \min (36 \text { min cada sessão) em } \\
\text { duas sessões. Os resultados foram } \\
\text { reavaliados } 12 \text { meses após o tratamento. }\end{array}$ \\
\hline Géis clareadores & $\begin{array}{l}\text { PH } 37,5 \% \text { e PH } 6 \% \text { (Polaoffice + 6\%, SDI, } \\
\text { Victoria, Australia). }\end{array}$ \\
\hline Resultados & $\begin{array}{l}\text { Concentrações baixas }(6 \%) \text { e tradicionais } \\
\text { foram eficazes e estáveis em um ano pós- } \\
\text { clareamento, embora sua eficácia tenha sido } \\
\text { estatisticamente diferente. }\end{array}$ \\
\hline Efeitos adversos & $\begin{array}{l}\text { O grupo tratado com } \mathrm{PH} 37,5 \% \text { apresentou } \\
\text { maior risco e intensidade de sensibilidade }\end{array}$ \\
\hline \multicolumn{2}{|l|}{ Kothari et al., 2020} \\
\hline País & Nova zelândia \\
\hline $\begin{array}{l}\text { Tipo de clareamento/ } \\
\text { Protocolo clínico }\end{array}$ & $\begin{array}{l}\text { Clareamento em consultório, caseiro e } \\
\text { associado. 105 pacientes foram divididos em } \\
\text { três grupos. G1: Procedimento caseiro, foi } \\
\text { utilizado PC a 10\%, 8h/dia, durante, 14 dias. } \\
\text { G2: procedimento em consultório, foi } \\
\text { empregado o PH a 37,5\%, em 1 sessão com } 3 \\
\text { aplicacóes de } 8 \text { minutos. G3: associação das } \\
\text { duas técnicas, seguindo os mesmos protocolos }\end{array}$ \\
\hline Géis clareadores & $\begin{array}{l}\text { PC } 10 \% \text { bleaching gel (Polanight, SDI, } \\
\text { Australia); PH } 37,5 \% \text { (Pola in-office + SDI, } \\
\text { Australia). }\end{array}$ \\
\hline Resultados & $\begin{array}{l}\text { O tratamento associado resultou em melhora } \\
\text { acentuada da cor quando comparado às duas } \\
\text { técnicas utilizadas individualmente. }\end{array}$ \\
\hline Efeitos adversos & $\begin{array}{l}\text { Baixos níveis de sensibilidade dentária nos } 3 \\
\text { grupos. }\end{array}$ \\
\hline \multicolumn{2}{|l|}{ Sutil et al., 2020} \\
\hline País & Brasil \\
\hline $\begin{array}{l}\text { Tipo de clareamento/ } \\
\text { Protocolo clínico }\end{array}$ & $\begin{array}{l}\text { Clareamento caseiro. } 80 \text { pacientes divididos } \\
\text { em dois grupos. G1: PC } 37 \% \text {; G2: PC } 10 \% \text {. Em } \\
\text { ambos os grupos foi realizado clareamento } \\
\text { por } 3 \text { semanas. 4h/dia para o grupo de } 10 \% \text { e } \\
30 \text { min/dia para o grupo de } 37 \% \text {. }\end{array}$ \\
\hline Géis clareadores & $\begin{array}{l}\text { PC } 10 \% \text { (Whiteness Perfect } 10 \% \text { carbamide } \\
\text { peroxide, FGM); PC a } 37 \% \text { (Whiteness Super } \\
37 \% \text { carbamide peroxide, FGM). }\end{array}$ \\
\hline Resultados & $\begin{array}{l}\text { O grupo PC a } 37 \% \text { apresentou clareamento } \\
\text { mais rápido que o grupo de } 10 \% \text {. No entanto, } \\
1 \text { mês após a conclusão, ambos os grupos } \\
\text { apresentaram clareamento equivalente. }\end{array}$ \\
\hline Efeitos adversos & $\begin{array}{l}\text { Baixos níveis de sensibilidade dentinária, } \\
\text { irritaçẫo gengival e não afetou saúde bucal }\end{array}$ \\
\hline
\end{tabular}

Rezende et al. ${ }^{5}$, ao testarem os efeitos do clareamento de consultório associado ao clareamento em caseiro (técnica combinada), apontaram a eficácia e a estabilidade ao longo de 12 meses após o tratamento. Tal resultado independeu da concentração do $\mathrm{PH}$ usado para a fase de estudo em consultório, ou seja, o PH a 
$20 \%$ e $35 \%$, utilizados, ambos trouxeram resultados semelhantes no efeito clareador. No entanto, o uso de um $\mathrm{PH}$ com concentração inferior para o clareamento em consultório resultou em menor risco e intensidade de SD. Resultados semelhantes foram obtidos por Machado et al. $^{18}$, ao afirmarem que a sessão em consultório prévia ao clareamento caseiro é um fator desencadeante para maior nível de SD, sem trazer benefícios efetivos no clareamento.

O estudo de Lima et al. ${ }^{19}$, fizeram uso do $\mathrm{PH}$ a $35 \%$ e a $15 \%$ em duas sessões de tratamento em consultório. Com isso, concluíram que o gel de maior concentração desempenhou melhor efeito clareador, entretanto, gerou maior risco e intensidade de SD. Já Martins et al. $^{20}$, constataram que a aplicação do $\mathrm{PH}$ a $38 \%$ pode ocorrer em um único momento de 40 minutos ou em dois de 20 minutos, sem influenciar no nível de clareamento, tampouco no de SD. Desse modo, a aplicação única de 40 minutos em 2 sessões foi o recomendado, haja vista a não necessidade de se renovar o gel.

No estudo de Loguercio et al. ${ }^{21}$, avaliouse a efetividade de géis clareadores com diferentes concentrações de $\mathrm{pH}$ (ácido e neutro). 54 voluntários das cidades de Ponta Grossa (Paraná, Brasil) e Santiago (Santiago, Chile) foram selecionados. Duas semanas antes dos procedimentos de clareamento, todos os voluntários receberam uma triagem e profilaxia dentária com pedra-pomes e água. Ao final do estudo, constatou-se que ambos os géis promoveram efeitos clareadores significativos, não havendo diferenças relevantes entre os grupos. Entretanto, no que tange a SD, o uso do gel ácido (Pola Office) produziu risco e intensidade significativamente maior do que 0 gel neutro.

Vildósola et al. $^{22}$, analisarem a efetividade do $\mathrm{PH}$ a $6 \%$ com dois diferentes protocolos, onde um grupo realizou única aplicação por 36 minutos e outro com três aplicações por 12 minutos, ambos em 2 sessões. Sendo assim, constatou-se que o nível de clareamento foi equiparável e, além disso, nos dois protocolos os pacientes relataram nível de SD leve. Tais resultados confluem com os de Bersezio et al. ${ }^{23}$, pois ao testaram a eficácia do $\mathrm{PH}$ a $6 \%$ concluíram que se trata de um agente efetivo, porém ao compará-lo com $\mathrm{O} \mathrm{PH}$ a $37,5 \%$ o nível de clareamento é significativamente inferior, e com o nível e intensidade de SD equiparável.

Os achados de Pinto et al. ${ }^{2}$, apontam que $\circ \mathrm{PH}$ tem eficácia nas concentrações de $6 \%, 7,5 \%$ e $10 \%$, haja vista que em todos os protocolos houveram estabilidade de cor após 12 meses de acompanhamento e a maioria dos indivíduos ficaram satisfeitos com o tratamento. Além disso, observou-se que o $\mathrm{PH}$ em maior concentração trouxe um efeito clareador mais rápido, no entanto, os pacientes relataram maior desconforto.

Os resultados de Chemin et al. $^{7}$ evidenciaram que $\mathrm{O} \mathrm{PH}$ tem eficácia nas concentrações de $4 \%$ e $10 \%$ e ambos apresentaram estabilidade de cor após um mês de acompanhamento. Observou-se que o $\mathrm{PH}$ em maior concentração trouxe um efeito clareador mais rápido, no entanto, os pacientes relataram maior risco e intensidade de SD. Para Cordeiro et al. ${ }^{24}$, o $\mathrm{PH}$ a $10 \%$ também é eficaz ao promover clareamento, independente da forma de administração, podendo ser utilizado em moldeiras convencionais ou descartáveis.

Ferraz et al. ${ }^{25}$, analisaram os efeitos do clareamento de consultório com peróxidos em baixas concentrações, sendo testados $\mathrm{O} \mathrm{PH}$ a $6 \%$ e $15 \%$. Desse modo, foi possível concluir que ambos os agentes foram eficazes no clareamento, no entanto, decorridos 6 meses do tratamento, 0 grupo que realizou 0 procedimento com o gel de maior concentração apresentou estabilidade de cor significativamente melhor. Outrossim, não houve diferenças importantes entre os grupos em relação à SD ou irritação gengival. Estay et al. ${ }^{26}$,também verificaram a eficácia do $\mathrm{PH}$ a $6 \%$ e atestaram que 0 produto de baixa concentração demonstrou resultados promissores, incluindo alto desempenho, eficácia, com sensibilidade pós-operatória muito baixa.

Os autores Zlataric et al. ${ }^{27}$ e Kothari et al. ${ }^{28}$, concluíram que a associação das técnicas traz benefícios reais no nível de clareamento, tratando-se de um procedimento eficaz, pois o procedimento em casa aumenta significativamente 0 clareamento quando realizado após sessão prévia em consultório. Vaez et al. ${ }^{8}$, inferem que uma sessão preliminar de consultório reduziu o tempo necessário para obter a cor satisfatória, porém aumenta-se consideravelmente o risco e o nível de SD.

Por fim, Sutil et al. ${ }^{29}$ analisaram apenas o agente clareador PC, nas concentrações de $10 \%$ e $37 \%$ e observaram que o uso de PC a $37 \% / 30 \mathrm{~min} /$ dia pode diminuir o tempo de uso da moldeira no protocolo domiciliar, pois os resultados obtidos foram equivalentes ao peróxido de carbamida a $10 \%$ utilizado $4 \mathrm{~h} / \mathrm{dia}$. DISCUSSÃO

Atualmente, o fator estético consiste em 
uma das principais razões para os pacientes que procuraram atendimento odontológico, sendo crescente a busca por um sorriso cada vez mais harmônico ${ }^{8}$. Segundo Pasquali et al ${ }^{30}$, a cor da estrutura dentária desempenha papel extremamente importante do ponto de vista estético, pois é fácil e rapidamente percebida, portanto, o escurecimento dental interfere negativamente na aparência dos dentes. Nessa perspectiva, Lucena et al. $^{31}$, afirmam que 0 paciente pode ter sua autoestima e bem-estar prejudicados por queixas em relação a estética do seu sorriso.

A pigmentação ocorre por meio da formação de estruturas quimicamente estáveis, o que provoca a instalação de pigmentos na coroa dentária. Além disso, a cor do dente é determinada de acordo com o trajeto que a luz percorre ao incidir em sua superfície, dependendo das características dele, a luz pode sofrer reflexão, transmissão, dispersão e absorção. Desse modo, quanto mais elevada a quantidade de pigmentos, maior a absorção da luz incidida $e$, consequentemente, há um escurecimento do dente ${ }^{3}$.

O surgimento de pigmentos ocorre na dependência de inúmeros fatores extrínsecos e intrínsecos. O manchamento extrínseco pode ser causado pela ingestão de alimentos com corantes, pelo uso excessivo do fumo, por acúmulo da placa bacteriana e utilização de alguns tipos de medicamentos. Sua remoção é, geralmente, simples e depende de uma boa higienização ou profilaxia feita em consultório odontológico ${ }^{32}$.

Em relação as alterações de origem intrínseca, as mesmas podem ocorrer devido a uma série de fatores: alterações na formação do dente, doenças ocorridas durante a gestação, trauma dental, mortificação pulpar, acesso inadequado à câmara pulpar, má utilização de fármacos e de materiais de preenchimento, fluorose e envelhecimento dos dentes ${ }^{4}$. Segundo Vieira et al. $^{3}$, em dentes vitais é natural e fisiológico o aumento da deposição gradual de dentina ao longo da vida, o que resulta em dentes amarelados ou acinzentados. A correção de tais pigmentos intrínsecos é feita por meio de tratamento clareador e/ou estético.

Nesse âmbito, o clareamento dental é de grande valia para obtenção de um sorriso mais claro. Trata-se de um procedimento cada vez mais conservador, seguro, de fácil execução e com bons resultados a longo prazo ${ }^{30}$. Para tal, diferentes técnicas e protocolos podem ser empregados pelo profissional, variando o tipo do gel clareador, concentração, modo e tempo de aplicação. Os diferentes protocolos podem ou não resultar em melhores efeitos clareadores e/ou menores efeitos colaterais ${ }^{33}$.

Existem três produtos básicos para realização do tratamento clareador, o Peróxido de Hidrogênio $(\mathrm{PH})$, Peróxido de Carbamida (PC) e o Perborato de Sódio (PS), este último exclusivo para dentes desvitalizados. ${ }^{34}$ Segundo Llena et al. ${ }^{6}$, o mecanismo de ação desses agentes clareadores para remoção dos pigmentos, envolve reação de oxidação, ocorrendo a conversão dos materiais orgânicos em dióxido de carbono $\left(\mathrm{CO}_{2}\right)$ e água, removendo, consequentemente, os pigmentos da estrutura dentária por difusão.

As técnicas do clareamento dental envolvem procedimentos realizados tanto em consultório como em casa, ou, ainda, a associação das duas formas. $\mathrm{Na}$ técnica realizada em consultório a concentração do gel é mais elevada ( $20 \%$ a $38 \%)$, já na técnica realizada em casa o produto costuma variar de $3 \%$ a $22 \%{ }^{35}$ Para a Associação Dental Americana, todas as três técnicas de clareamento citadas anteriormente são eficazes, mas os resultados variam de acordo com o nível de pigmentação, idade do paciente, a concentração do agente ativo, o tempo e frequência do tratamento.

O clareamento dental caseiro supervisionado é realizado com moldeiras dentárias individuais e é uma das abordagens mais comumente usadas para modificar a cor do dente. As principais vantagens desta técnica estão relacionadas à facilidade de uso pelo paciente, tempo clínico reduzido e um nível comparativamente semelhante ou inferior de sensibilidade dentária e irritação gengival durante o tratamento em relação aos métodos de atendimento usando altas concentrações de peróxidos. Para isso, pode ser utilizado tanto o $\mathrm{PH}$ quanto o PC. ${ }^{36}$

Segundo Cordeiro et al. $^{24}$, desde a introdução da técnica do clareamento caseiro usando peróxido de carbamida a $10 \%$ durante a noite, resultados de sucesso a longo prazo foram relatados, o que condiz com os achados encontrados nesta pesquisa (Carlos et al. ${ }^{16}$; Machado et al $^{18}$; Rezende et al. ${ }^{5}$; Vaez et al. ${ }^{8}$; Kothari et al. ${ }^{28}$; Sutil et al. ${ }^{29}$ ). Mendes et al. ${ }^{37}$, apontam que o $\mathrm{PH}$ e o PC são eficazes quando administrados em concentrações de $4 \%$ a $16 \%$ com aplicações diárias que podem variar de 7 a 14 dias, sendo o tempo de aplicação do $\mathrm{PH}$ de 1 a 4 horas e do PC de 4 a 8 horas. Além disso, concluem que os géis com menores concentrações provocam menos sensibilidade dentária e irritação gengival.

Já o clareamento dental em consultório 
também é um método estético amplamente utilizado. Um procedimento químico que permite a obtenção de um efeito clareador mais rápido, além de dispensar o uso das moldeiras de clareamento, o que é incomodo para alguns pacientes $^{38}$. Para Alves et al. ${ }^{39}$, a utilização do peróxido de hidrogênio e do peróxido de carbamida, ambos a $35 \%$, apresenta uma excelente eficácia e qualidade do clareamento, sendo capaz de produzir menor irritação gengival.

Entretanto, a utilização de altas concentrações de peróxido de hidrogênio (25\% $-35 \%$ ) para procedimentos de clareamento em consultório, é apontada como um fator que pode aumentar a incidência e a intensidade de sensibilidade dentária, bem como a de irritação gengival $^{40}$.

A hipótese mais aceita para explicar a alta prevalência de sensibilidade dentária é a rápida difusão do $\mathrm{PH}$ e produtos de degradação para dentro da câmara pulpar, como também a reação desses produtos pode atingir os tecidos moles e causar lesões como irritação gengival, queimaduras e ulceração ${ }^{41}$.

A hipersensibilidade dentinária é caracterizada por uma dor súbita, aguda e de curta duração decorrentes de estímulos térmicos, químicos, tácteis e evaporativos, que não pode ser atribuída outra patologia dentária $^{42}$. Não é raro, pacientes submetidos a tratamentos clareadores relatarem sensações dolorosas nos dentes tratados, o que pode ser resultado do aumento da permeabilidade do esmalte, com uma possível difusão do peróxido até a polpa ${ }^{43}$. Em concordância com essa afirmação, os dados desta pesquisa evidenciaram que a sensibilidade dentária é um achado comum nos pacientes que realizam o clareamento dental.

Geralmente, os casos de sensibilidade dentária duram, no máximo, até 4 dias após o tratamento clareador, e é mais comum em procedimentos que utilizam $\mathrm{PH}$ em altas concentrações e/ou de fontes de luz. ${ }^{44}$ Outrossim, para Reis ${ }^{11}$, pacientes com histórico de sensibilidade dentária tem maior probabilidade de desenvolver hipersensibilidade pós-operatória ao clareamento, o que pode estar associado a presença de retrações gengivais, trincas e desgaste de esmalte e baixo limiar de dor.

Nos estudos de Chemin et al. ${ }^{17}$ e Estay et al. ${ }^{26}$, observou-se que quando utilizadas altas concentrações, as probabilidades de ocorrerem maiores níveis de sensibilidade pulpar e periodontal aumentam. Para Vaez et al. ${ }^{8}$, a técnica associada acarretou maior nível de SD, quando comparada apenas à técnica caseira supervisionada.

Ainda em relação aos efeitos colaterais, Monteiro et al. $^{45}$, relataram que em torno de $80 \%$ de todos os pacientes apresentam sensibilidade e irritação gengival, pelo menos um dia durante o tratamento. Segundo Lima et al. $^{46}$, o protocolo com PC a $35 \%$, resulta em aumento de sensibilidade dentária e outros efeitos adversos quando comparado ao $\mathrm{PH}$ a $20 \%$, mesmas conclusões apontadas por Rezende et al. ${ }^{5}$, ao testarem o $\mathrm{PH}$ a $35 \%$ e $\mathrm{PH}$ a $20 \%$.

$\mathrm{Na}$ contramão desses estudos, os achados de Kothari et al. ${ }^{28}$, concluíram que 0 $\mathrm{PH}$ a $37,5 \%$ não causou maior nível de SD do que o PC a $10 \%$, onde ambos os protocolos apresentaram nível leve de desconforto. O estudo de Lima et al. ${ }^{19}$, fez uso do $\mathrm{PH}$ a $35 \%$ e a $15 \%$ em duas sessões de tratamento em consultório, e com isso concluíram que o gel de maior concentração desempenhou melhor efeito clareador, entretanto, gerou maior risco e intensidade de SD. Já Martins et al. $^{20}$, constataram que a aplicação do $\mathrm{PH}$ a $38 \%$ tanto em aplicação única de 40 minutos como em duas de 20 minutos, não influenciou no nível de clareamento nem no de SD.

CONCLUSÃO

O clareamento dental caseiro supervisionado e o de consultório configuram tratamentos simples, eficientes, seguros e econômicos, entretanto, apresentam riscos que podem ser eliminados ou minimizados. Desse modo, para o emprego de um protocolo clinicamente eficaz é imprescindível um correto diagnóstico, já que o tipo de pigmento influencia sobremaneira no protocolo a ser utilizado. Para isso, é fundamental conhecer a etiologia da mancha a fim de estabelecer o tipo do gel, a sua concentração, o tempo e modo de aplicação adequados.

É certo que os géis clareadores comumente utilizados, apesar de apresentarem mecanismos de ação diferentes, são eficazes, quando corretamente administrados. Com isso, foi possível constatar que agentes de clareamento com concentrações mais baixas geram menos efeitos adversos, porém, para se alcançar a cor almejada e com maior estabilidade, a maioria dos clínicos estende o tempo de uso ou fazem a associação das técnicas. Nesse contexto, a hipersensibilidade dentária e a irritação gengival pós-tratamento são relatos constantes, e foram, na maioria dos estudos, os mais frequentes.

Em suma, vale ressaltar a importância do conhecimento por parte do profissional no 
que tange ao correto diagnóstico, a real necessidade do paciente $e$ as limitações encontradas em cada técnica, assegurando, dessa forma, um tratamento com bons resultados de clareamento, de estabilidade de cor ao longo do tempo e com ausência ou baixo índice de efeitos adversos.

REFERÊNCIAS

1. Kwon SR, Wertz EP. W. Review of the mechanism of tooth whitening. J Esthet Restor Dent. 2015;27(5):240-57.

2. Pinto MM, Gonçalves MLL, Mota ACC, Deana AM, Olivan SR, Bortoletto C, et al. Controlled clinical trial addressing teeth whitening with hydrogen peroxide in adolescents: a 12-month follow-up. Clinics. 2017;72(3):161-70.

3. Vieira APSB, Leitão AS, Patrício CEG, Cerqueira FS. Consequências do clareamento em dentes vitais e na saúde geral do paciente. Campo do Saber. 2018;4(5):33-47.

4. Joiner $A$. The bleaching of teeth: a review of the literature. J Dent. 2006; 34(7):412-419.

5. Rezende M, Siqueira SH, Kossatz S. Clareamento dental - efeito da técnica sobre a sensibilidade dental e efetividade. Rev Assoc Paul Cir Dent. 2014;68(3):208-12.

6. Llena C, Villanueva A, Mejias E, Forner L. Bleaching efficacy of at home $16 \%$ carbamide peroxide A long-term clinical follow-up study. J Esthet Restor Dent. 2020;32(1):12-8.

7. Chemin K, Rezende M, Loguercio AD, Reis A, Kossatz S. Effectiveness of and Dental Sensitivity to At-home Bleaching With $4 \%$ and 10\% Hydrogen Peroxide: A Randomized, Triple-blind Clinical Trial. Oper Dent. 2018; 43(3):232-40.

8. Vaez SC, Correia A, Santana TR, Santana M, Peixoto AC, Leal PC et al. Is a Single Preliminary Session of In-office Bleaching Beneficial for the Effectiveness of At-home Tooth Bleaching? A Randomized Controlled Clinical Trial. Oper Dent. 2019;4(44):180-89.

9. Marson FC, Sensi LG, Araujo FO, Monteiro Junior S, Araujo E. Avaliação clínica do clareamento dental pela técnica caseira. Rev Dental Press Estét. 2007;2(4):50-60.

10. Briso ALF, Rahal V, Gallinari M. O. Análise do clareamento dental caseiro realizado com diferentes produtos: relato de caso. Rev Odontol Araçatuba. 2014;35(1):49-54.

11. Reis A, Loguercio AD. Materiais Dentários Restauradores Diretos - dos Fundamentos à Aplicação Clínica. São Paulo: Santos; 2007.

12. Moghadam FV. The degree of colorchange, rebound effect and sensitivity of bleached teeth associated with at-homeand power bleaching techniques: A randomized clinical trial. Eur $\mathrm{J}$ Dent. 2013;7(4):405-11.
13. Francci C. Clareamento dental-técnicas e conceitos atuais. Rev Assoc Paul Cir Dent. 2010;64(1):78-89.

14. Kose C, Calixto AL, Bauer JR, Reis A, Loguercio AD. Comparison of the Effects of Inoffice Bleaching Times on Whitening and Tooth Sensitivity: A Single Blind, Randomized Clinical Trial. Oper Dent. 2016;41(2):138-45.

15. Bacaksiz A, Tulunoglu O, Tulunoglu I. Efficacy and Stability of Two in-Office Bleaching Agents in Adolescents: 12 Months Follow-Up. J Clin Pediatr Dent. 2016;40(4):269-73.

16. Carlos NR, Bridi EC, Amaral F, França F, Turssi CP, Basting RT. Efficacy of Home-use Bleaching Agents Delivered in Customized or Prefilled Disposable Trays: A Randomized Clinical Trial. Oper Dent. 2017;42(1):30-40.

17. Chemin K, Rezende M, Milan FM, Dantas TB, Gomes KDN, Kossatz S. Clinical Evaluation of 10\% Hydrogen Peroxide on Tooth Sensitivity and Effectiveness in at Home Dental Bleaching. J Contemp Dent Pract. 2018;19(11):1376-80.

18. Machado LS, Anchieta RB, dos Santos PH, Briso AL, Tovar $\mathrm{N}$, Janal $\mathrm{MN}$ et al. Clinical Comparison of At-Home and In-Office Dental Bleaching Procedures: A Randomized Trial of a Split-Mouth Design. Int $\mathrm{J}$ Periodontics Restorative Dent. 2016;36(2):251-60.

19. Lima RRM. Avaliação clínica da efetividade de dois sistemas de clareamento dental de consultório após 10 meses - série de casos. Rev Uningá. 2016;47:51-6.

20. Martins I, Onofre S, Franco N, Martins LM, Montenegro A, Arana-Gordillo LA et al. Effectiveness of In-office Hydrogen Peroxide With Two Different Protocols: A Two-center Randomized Clinical Trial. Oper Dent. 2018; 43(4):353-61 .

21. Loguercio $A D$, Servat $F$, Stanislawczuk $R$, Mena-Serrano A, Rezende M, Prieto MV, et al. Effect of acidity of in-office bleaching gels on tooth sensitivity and whitening: a two-center double-blind randomized clinical trial. Clin Oral Investig. 2017;21(9):2811-18.

22. Vildósola $P$, Vera $F$, Ramírez J, Rencoret $J$, Pretel $\mathrm{H}$, Oliveira $\mathrm{OB} \mathrm{Jr}$, et al. Comparison of Effectiveness and Sensitivity Using Two InOffice Bleaching Protocols for a 6\% Hydrogen Peroxide Gel in a Randomized Clinical Trial. Oper Dent. 2017;42(3):244-52.

23. Bersezio C, Estay J, Jorquera G, Peña $M$, Araya C, Angel P, et al. Effectiveness of Dental Bleaching With $37.5 \%$ and $6 \%$ Hydrogen Peroxide and Its Effect on Quality of Life. Oper Dent. 2019; 44(2):146-55.

24. Cordeiro D, Toda C, Hanan S, Arnhold LP, Reis $A$, Loguercio $A D$ et al. Clinical Evaluation of Different Delivery Methods of At-Home Bleaching Gels Composed of 10\% Hydrogen Peroxide. Oper Dent. 2019;44(1):13-23. 
25. Ferraz NKL, Nogueira LC, Neiva IM, Ferreira RC, Moreira AN, Magalhães CS. Longevity, effectiveness, safety, and impact on quality of life of low-concentration hydrogen peroxides inoffice bleaching: a randomized clinical trial. Clin Oral Investig. 2019;23(5):2061-70.

26. Estay J, Angel $P$, Bersezio $C$, Tonetto $M$, Jorquera G, Peña $M$ et al. The change of teeth color, whiteness variations and its psychosocial and self-perception effects when using low vs. high concentration bleaching gels: a one-year follow-up. BMC Oral Health. 2020;20(1):255.

27.Zlatarić D, Žagar M, Illeš D. A clinical study assessing the short-term efficacy of combined in-office/at-home whitening treatment. J Esthet Restor Dent. 2019;31(2):140-46.

28. Kothari S, Jum'ah AA, Gray AR, M Lyons K, Yap M, Brunton PA. A randomized clinical trial investigating three vital tooth bleaching protocols and associated efficacy, effectiveness and participants' satisfaction. J Dent. 2020;95:103322.

29. Sutil E, da Silva KL, Terra RMO, Burey A, Rezende M, Reis A et al. Effectiveness and adverse effects of at-home dental bleaching with $37 \%$ versus $10 \%$ carbamide peroxide: $A$ randomized, blind clinical trial. J Esthet Restor Dent. 2020. doi: 10.1111/jerd.12677.

30. Pasquali EL, Bertazzo CA, Anziliero L. Estudo dos efeitos do clareamento dental sobre 0 esmalte: uma revisão das evidências para a indicação clínica. Perspectiva. 2014;38(141): 99-108.

31. Lucena MTL. Clareamento interno em dentes desvitalizados com a técnica walking bleach relato de caso. Uningá Review.2015;24(1):33-9.

32. Touati, B. Sistemas Cerâmicos Atuais. In: Odontologia Estética e Restaurações Cerâmicas. São Paulo: Santos; 2000.

33. Kinn PW, Barnes DM, Romberg E, Peterson K. A clinical evaluation of 10 percent vs 15 percent carbamide peroxide tooth-whitening agents. $\mathrm{J}$ Am Dental Assoc. 2000;131(10):1478-84.

34. Navarro MFL, Mondelli RFL. Riscos com o clareamento dental. In: Cardoso RJA, Gonçalves EAN. Odontologia estética. São Paulo: Artes Médicas; 2002.

35. Bruzell EM. .Side effects of external tooth bleaching therapy with activation by heat, light or laser: a systematic review. Dent Mater. 2007; 23(5):586-96.

36. Nunes Junior AP. Clareamento de dentes vitais: 0 estado da arte [monografia]. Florianópolis: Universidade Federal de Santa Catarina; 2001.

37. Mendes JL, Vasconcelos RG, Vasconcelos MG. Avaliação dos diferentes protocolos de clareamento dental caseiro (supervisionado). Salusvita. 2020; 39(3):797-809.
38. Sulieman AM. An overview of tooth-bleaching techniques: chemistry, safety and efficacy. Periodontol 2000. 2008;48(1):148-69.

39. Alves LN, Vasconcelos MG, Vasconcelos RG. Análise dos diferentes protocolos e técnicas de clareamento dentário em consultório: uma revisão de literatura. Salusvita. 2020;39(3): 811-28.

40.Carey CM. Tooth whitening: what we now know. J Evid Based Dent Pract. 2014; 14(Suppl):70-6.

41. Markowitz K. Pretty painful: Why does tooth bleaching hurt. Med Hypotheses. 2010;74(5): 835-40.

42. Shintome LK, Umetsubo LS, Nagayassu MP, Jorge ALC, Gonçalves SEP, Torres CRG. Avaliação clínica da laserterapia no tratamento da hipersensibilidade dentinária. Ciênc odontol bras. 2007;10(1):26-36.

43. Henrique DBB. Os Pricipais Efeitos Colaterais Do Clareamento Dentário: Como Amenizá-Los. Salusvita. 2017;36(1):141-55.

44.Dahl JE, Pallesen U. Tooth Bleaching--A Critical Review of the Biological Aspects. Crit Rev Oral Biol Med. 2003;14(4):292-304.

45. Monteiro MJF, Lindoso JBC, Conde NCO, Silva LMS, Loguercio AD, Pereira JV. Evaluation of the genotoxic potential of different delivery methods of at-home bleaching gels: a singleblind, randomized clinical trial. Clin Oral Investig. 2019;23(5):2199-206.

46. Lima SNL, Ribeiro IS, Grisotto MA, Fernandes ES, Hass V, de Jesus Tavarez RR et al. Evaluation of several clinical parameters after bleaching with hydrogen peroxide at different concentrations: A randomized clinical trial. J Dent. 2018;68:91-7.

\section{CONFLITO DE INTERESSES}

Os autores declaram não haver conflitos de interesse

\section{AUTOR PARA CORRESPONDÊNCIA}

Rodrigo Gadelha Vasconcelos
Universidade Estadual da Paraíba
Av. Coronel Pedro Targino,
58233-000 Araruna-PB, Brasil
Tel: (83) 3373-1040
e-mail: rodrigogadelhavasconcelos@yahoo.com.br

Submetido em 18/05/2021 Aceito em 16/07/2021 